\title{
Behaviour of plated structures subjected to blast loading
}

\author{
Vegard Aune ${ }^{\mathrm{a}}$, Tore Børvik, and Magnus Langseth \\ Structural Impact Laboratory (SIMLab), Centre for Research-based Innovation (CRI), Department of Structural \\ Engineering, Norwegian University of Science and Technology (NTNU), NO-7491 Trondheim, Norway
}

\begin{abstract}
An experimental investigation using a new shock tube facility to study blast-load effects on thin aluminium plates is presented. The shock tube is designed to expose materials and structures to extreme loading conditions, such as accidental explosions or terrorist attacks. The intensity of the loading in the present study was determined by the initial conditions of the compressed gas, i.e. volume and pressure, and the resulting loading on the target plate was compared to experimental data from explosive detonations found in the literature. The square plates were manufactured from a low-strength aluminium alloy and had an exposed area of $0.3 \times 0.3 \mathrm{~m}^{2}$. Piezoelectric pressure sensors were used for pressure recordings and synchronized with two high-speed cameras operating at a frame rate of $21,000 \mathrm{fps}$ in a stereoscopic setup to capture the dynamic response using a threedimensional digital image correlation (3D-DIC) technique. The experiment showed that the shock tube is capable of recreating a loading similar to that of an unconfined far-field airblast, and worked as an easily controllable alternative to explosive detonations when studying the dynamic response of structures subjected to blast loading.
\end{abstract}

\section{Introduction}

Protection of engineering structures against blast loading has received a lot of attention in recent years [1-3]. Depending on the blast intensity the material and structural behaviour may become significantly different from their quasi-static behaviour if inertia and strain-rate effects become dominant. This results in the need to consider these effects in the design of protective structures involving blast events.

Due to the complexity in both the explosive loading and the resulting structural response, advanced numerical techniques are necessary for sufficient insight in the structural component optimization. However, before using such computational methods their performance should be validated in terms of reliability, robustness and effectiveness in predicting both blast loading and structural response. By its very nature experimental validation is ideal as it represents the actual physics of the problem, and controllable small-scale experiments could therefore be used to validate the computational methods and improve the understanding of the response of structures and materials under extreme loading situations (such as explosions). It may seem inviting to use scaled explosive detonations in such small-scale experiments. However, experimentation involving high-explosives may be hazardous and involves significant legal responsibilities which makes high-explosives less ideal for research purposes. An alternative to explosive detonations is the shock tube technique where pressure loading is generated by a rapid release of high-pressurized gas which creates a uniform shock wave propagating down a tube towards a test specimen. The loading is well defined by the initial conditions in the pressure chambers, and this technique

${ }^{a}$ Corresponding author: vegard. aune $@ n t n u . n o$ serves as an easily controllable and repeatable alternative to explosive detonations. Shock tube experiments also avoid the complexity of ground reflections, light flashes, fireballs and other potential challenges related to highexplosive detonations.

The objective of this study is to investigate the performance of a new shock tube facility in recreating a loading similar to that from a spherical airblast, so that the shock tube can be used as an alternative to explosive detonations when studying the dynamic response of aluminium plates subjected to blast loading. This is done by comparing the pressure time-history from the shock tube experiment to experimental data from explosive detonations found in the literature $[4,5]$. The dynamic response of the plate was studied using pressure measurements and two highspeed cameras in a stereoscopic setup, and the deformation field was found using three-dimensional digital image correlation (3D-DIC).

\section{Experimental setup}

\subsection{Material}

The $0.8 \mathrm{~mm}$ thick aluminium plate was manufactured from a low-strength, strain-hardened and cold-rolled sheet of the alloy EN AW 1050A-H14 produced by Hindalco Industries Ltd. This is $99.6 \%$ pure aluminium subjected to annealing before work hardened by rolling. The material is often used for sheet work where high mechanical properties are not required. The nominal chemical composition is provided in Table 1, while the nominal yield and tensile strengths were given by the producer to be $110 \mathrm{MPa}$ and $116 \mathrm{MPa}$, respectively. 


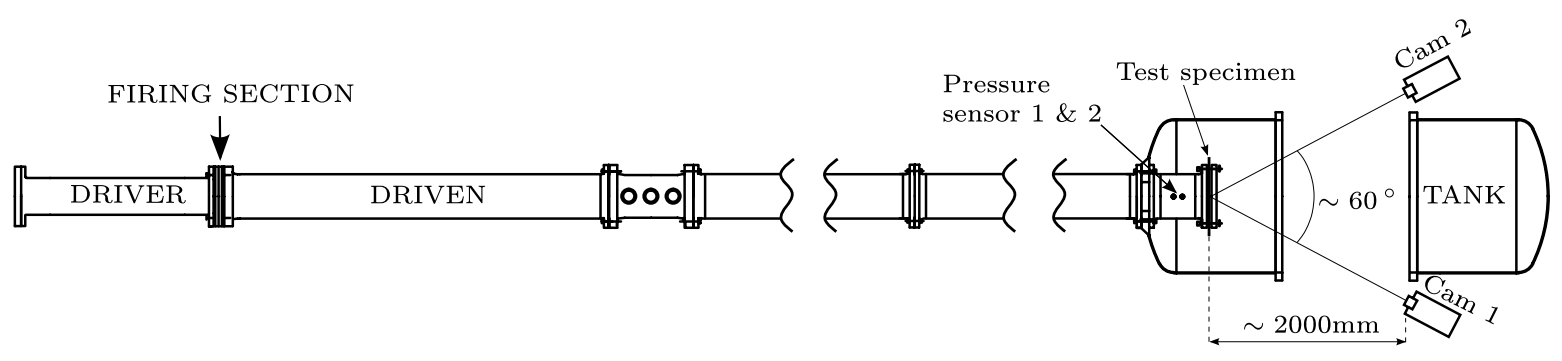

(a) Sketch of experimental setup (seen from above)

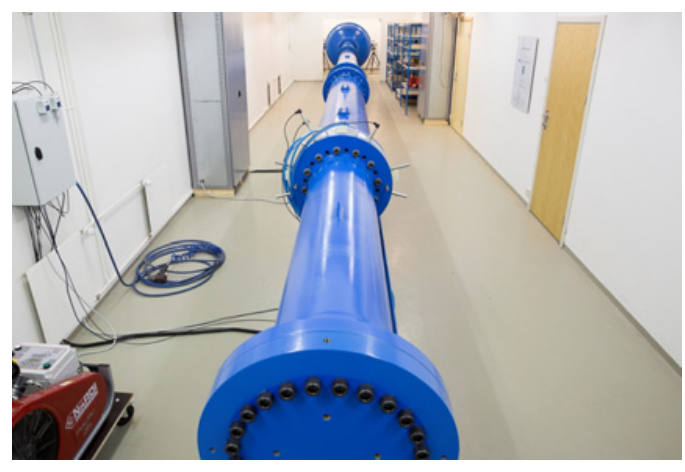

(b) Picture of the shock tube (seen from the driver)

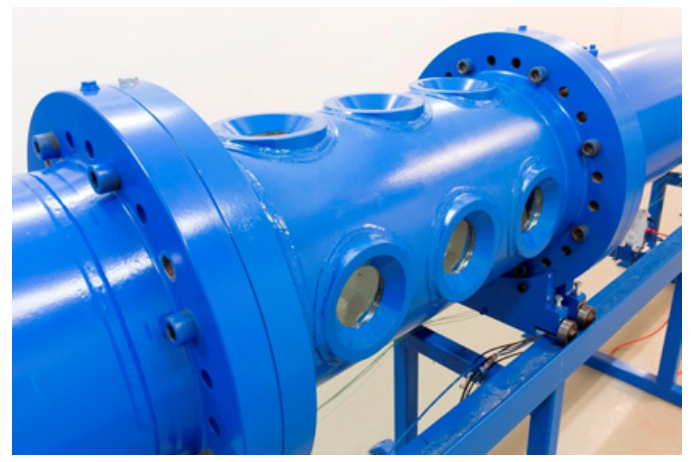

(d) Picture of the window section

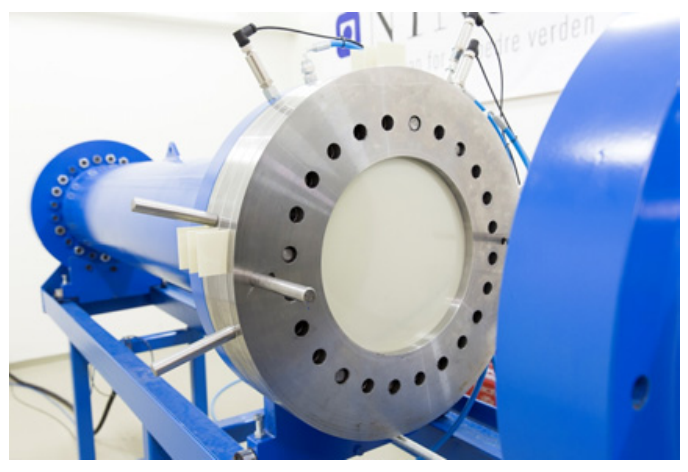

(c) Picture of the firing section

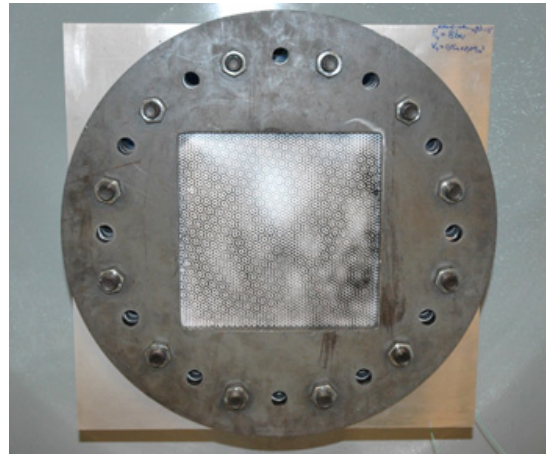

(e) Picture of clamping and DIC speckle pattern

Figure 1. Experimental setup in the SIMLab Shock Tube Facility. The speckle pattern is seen from the cameras.

Table 1. Chemical composition of EN AW 1050A-H14 (in wt.\%).

\begin{tabular}{ccccc}
\hline $\mathrm{Si}$ & $\mathrm{Fe}$ & $\mathrm{Cu}$ & $\mathrm{Mn}$ & $\mathrm{Mg}$ \\
\hline 0.100 & 0.280 & 0.003 & 0.010 & 0.002 \\
\hline $\mathrm{Zn}$ & $\mathrm{Ti}$ & $\mathrm{Cr}$ & $\mathrm{Al}$ & \\
\hline 0.002 & 0.009 & 0.002 & Rest & \\
\hline
\end{tabular}

\subsection{Shock tube experiment}

The shock tube experiment was performed in the new shock tube facility at the Structural Impact Laboratory, NTNU (Fig. 1). The overall length of the tube is $18.275 \mathrm{~m}$ and is made from $\mathrm{P} 355 \mathrm{NH}$ which is a stainless steel designed for pressure purposes according to EN 134451:2009. The high-pressure chamber of the shock tube (called driver in Fig. 1a and shown in Fig. 1b) is manufactured with a total length of $2.02 \mathrm{~m}$ and an inner diameter of $0.331 \mathrm{~m}$ where the internal wall is dull polished to obtain a smooth surface. The driver is followed by the firing section which consists of two intermediate pressure chambers that can be separated by membranes (Fig. 1c). This enables the total pressure difference to be achieved stepwise. The experiment starts by filling the driver and firing section with pressurized air, and the pressure in the intermediate chambers is operated such that the desired pressure is obtained in the driver. A LABVIEW-based program has been developed and solenoid valves (ASCO Series 223) have been installed on the gas-filling lines to control the filling procedure, making the process fully automated based on the signals given by the pressure sensors (BAUMER PBMN-24B31) mounted to the driver and intermediate pressure chambers. The rupture of the membranes is initiated by controlled venting of the intermediate pressure closest to the driver, using two solenoid valves (ASCO Series 223). This ensures a controlled rupture of the membranes.

The sudden release of the high pressure generates a shock wave propagating down the low-pressure chamber (called driven in Fig. 1a). The inner cross-section in the driven section starts with a transition from circular to a 
square cross-section, where an epoxy material is used to obtain a smooth surface and square cross-section of $0.3 \times$ $0.3 \mathrm{~m}^{2}$ inside the surrounding tube. The epoxy material works as a practically incompressible material while the surrounding tube ensures the structural strength. The square cross-section downstream the firing section was chosen to accommodate plane parallel windows (Fig. 1d) which simplifies the use of optical techniques, and enables the installation of test objects in threaded holes in the tube floor.

The maximum working pressure of the driver section is limited to $17 \mathrm{MPa}$ while the driven section, window section and dump tank are limited to $10 \mathrm{MPa}, 5 \mathrm{MPa}$ and $1.4 \mathrm{MPa}$, respectively. All respective parts of the facility were tested at a pressure $45 \%$ higher than the working pressure for a few minutes to ensure sufficient strength for routine use.

The experiment in the present work used aluminium inserts of diameter $0.33 \mathrm{~m}$ to reduce the initial volume in the driver to $23.2 \mathrm{dm}^{3}$, and it was sufficient with two Melinex membranes of $0.5 \mathrm{~mm}$ separated by only one intermediate pressure in the firing section. The high pressure was released when the overpressure in the driver section was $802.6 \mathrm{kPa}$, while the driven section was operated at ambient pressure $(100.13 \mathrm{kPa})$ and temperature $\left(20.5^{\circ} \mathrm{C}\right)$ before releasing the shock wave. The square plate specimen with dimensions of $0.625 \times 0.625 \times 0.0008 \mathrm{~m}^{3}$ had an exposed area of $0.3 \times 0.3 \mathrm{~m}^{2}$, and was clamped to the end flange of the tube using M24 bolts and a clamping frame in an attempt to achieve fixed boundary conditions (Fig. 1e).

Piezoelectric pressure sensors (Kistler 603B), corresponding charge amplifiers (Kistler 5064) and data acquisition system from National Instruments (NI USB6356) were used to measure the pressure downstream the firing section with a samling frequency of $500 \mathrm{kHz}$ during the experiments. The pressure transducers were placed in threaded adapters (Kistler 6501) and flush mounted in the tube wall, measuring the pressure behind the incident and reflected shock wave $29.5 \mathrm{~cm}$ (sensor 2) and $39.5 \mathrm{~cm}$ (sensor 1) upstream of the test specimen (see Fig. 1a). The delay in time of arrival of the shock wave at the pressure sensors was used to determine shock wave velocity and Mach number. A thin layer of insulating silicone (Kistler 1051) was used to shield the pressure transducers against heat transfer from the shock wave since the sensors are only designed for temperatures up to $200^{\circ} \mathrm{C}$.

\subsection{DIC measurements}

The plate was painted with a speckle pattern (Fig. 1e) and a three-dimensional digital image correlation (3D-DIC) analysis was conducted using a stereovision setup with two Phantom v1610 high-speed cameras. The separation angle between the optical axes of the cameras was approximately $60^{\circ}$ (see Fig. 1a). The recording rate was chosen to $21,000 \mathrm{fps}(21 \mathrm{kHz})$ with an image resolution of $896 \times 800$ pixels and 12-bit grey level digitization. An independent data acquisition system from National Instruments (NI USB-6356) was used to synchronize the pressure recordings with the images from the high-speed cameras. The camera calibration and image analysis were carried out in a post-processing phase using an in-house
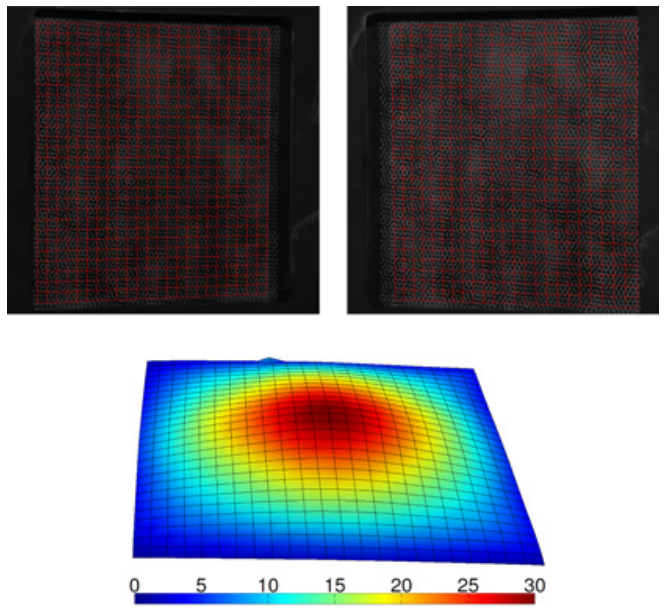

Figure 2. Results from 3D-DIC for the shock tube experiment. The colour scaling on the 3D-model indicates out-of-plane displacement (in $\mathrm{mm}$ ).

DIC code. The resulting residual root-mean-square (RMS) from the calibration was calculated to be approximately $0.22 \mathrm{~mm}$ ( $\sim 0.5$ pixels $)$ for both cameras.

Figure 2 shows an example of recorded images using the two synchronized high-speed cameras with the resulting DIC meshes plotted on top. The top images show two matching images from camera 1 (top-left) and camera 2 (top-right). The corresponding 3D model from DIC is presented in the lower image.

\section{Experimental results}

\subsection{Material tests}

Tensile tests on dog-bone specimens from the base material with clamped ends were performed to determine the quasi-static behaviour of the material while the dynamic material behaviour may be found in the literature $[6,7]$. The tests were performed in a Zwick/Roell Z030 testing machine at a constant deformation rate of $2.1 \mathrm{~mm} / \mathrm{min}$. This corresponds to an initial strain rate of $\dot{\varepsilon}=5 \times 10^{-4} \mathrm{~s}^{-1}$ for a gauge length of $70 \mathrm{~mm}$. Three parallel tests were performed in three different directions $\left(0^{\circ}, 45^{\circ}\right.$ and $\left.90^{\circ}\right)$ with respect to the rolling direction of the plate. Two-dimensional DIC was used to measure the displacement field and synchronized with the force measured by the hydraulic test machine at a sampling rate of $4 \mathrm{~Hz}$.

The results are plotted as nominal stress-strain curves in Fig. 3. It is seen that the material is slightly anisotropic both in flow stress and elongation to failure.

\subsection{Shock tube performance}

Figure 4 shows the measured pressure time-history at the respective sensors, while Table 2 gives the characteristic loading parameters for the sensor closest to the target plate (sensor 2) and the maximum out-of-plane displacement of the centre point $d_{z, \max }$. The reported blast wave parameters are limited to those related to the positive phase, i.e. the peak reflected overpressure $p_{r, \max }$, positive duration $t_{+}$ 


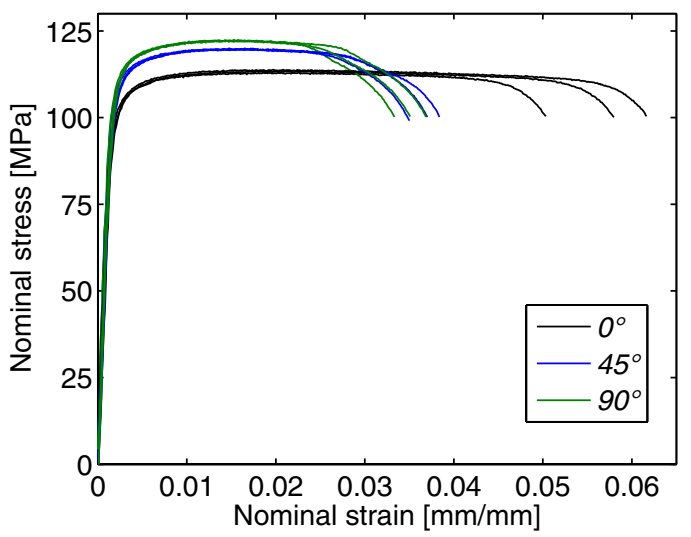

Figure 3. Nominal stress-strain curves from uniaxial tensile tests at three different loading directions.

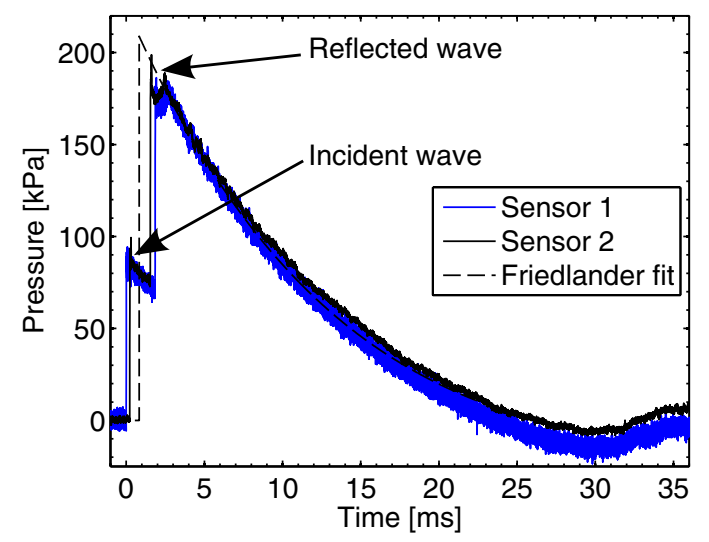

Figure 4. Pressure time-history from the experiment.

and impulse $I_{+}$. The impulse was found by numerical integration of the pressure curve during the positive duration at the selected sensor. The time of arrival of the incident shock wave at the first pressure transducer (sensor 1) was used as the starting point for the recordings in the experiment $(t=0)$.

A commonly used approximation to describe the pressure time-history for blast loading on a continuous format is the Friedlander equation [8], i.e.

$$
p(t)=p_{r}\left(1-\frac{t}{t_{+}}\right) \exp \left(\frac{-b t}{t_{+}}\right) .
$$

Curve fitting using the Levenberg-Marquardt algorithm in MATLAB gives the corresponding parameters of the Friedlander equation, and the reflected overpressure $p_{r, \max }$ at the plate was estimated to be $208.8 \mathrm{kPa}$ and the decay coefficient $b$ was determined to be 1.26 . This is plotted as a dotted line in Fig. 4 indicating the actual loading on the plate, and that the pressure loading in the shock tube follows the same exponential decay as an explosive detonation.

The difference in time of arrival at sensor 1 and 2 was used to determine the velocity $U_{s}$ of the incident shock wave to be $446.4 \mathrm{~m} / \mathrm{s}$, which corresponds to a Mach number $M_{s}$ of 1.3 . Knowing the shock velocity the ideal gas theory may be used to calculate the incident and reflected pressures. The derivation of these expressions is found in the literature (see e.g. [9]) and is therefore only
Table 2. Experimental results.

\begin{tabular}{cccccc}
\hline Test & $\begin{array}{c}p_{r, \max } \\
{[\mathrm{kPa}]}\end{array}$ & $\begin{array}{c}t_{+} \\
{[\mathrm{ms}]}\end{array}$ & $\begin{array}{c}I_{+} \\
{[\mathrm{kPa} \cdot \mathrm{ms}]}\end{array}$ & $\begin{array}{c}t_{+} / T_{n} \\
{[-]}\end{array}$ & $\begin{array}{c}d_{z, \max } \\
{[\mathrm{mm}]}\end{array}$ \\
\hline Shock tube & 208.8 & 25.4 & 1816.9 & 1.9 & 29.5 \\
\hline
\end{tabular}

briefly repeated below. The pressure behind the incident shock wave $p_{2}$ is given as

$$
p_{2}=p_{1}\left(1+\frac{2 \gamma_{1}}{\gamma_{1}+1}\left(M_{s}^{2}-1\right)\right)
$$

where $p_{1}$ is the initial pressure in the driven section and $\gamma_{1}$ is the corresponding ratio of specific heat at constant pressure to that at constant volume for the low-pressure gas. The Mach number $M_{s}$ is the ratio of the incident shock wave velocity $U_{s}$ to the speed of sound $a_{1}=$ $\sqrt{\gamma_{1} R T_{1} / m_{1}}$ in the undisturbed low-pressure gas, where $T_{1}$ is the measured initial temperature (in Kelvin), $m_{1}$ is the molecular weight and $R$ is the universal gas constant.

The pressure $p_{5}$ behind the reflected shock wave from a rigid wall can be calculated as

$$
p_{5}=p_{2}\left(\frac{\left(\alpha_{1}+2\right)\left(p_{2} / p_{1}\right)-1}{\left(p_{2} / p_{1}\right)+\alpha_{1}}\right)
$$

where the relation $\alpha_{1}=\left(\gamma_{1}+1\right) /\left(\gamma_{1}-1\right)$ is introduced for simplicity. Thus, using the relations from the ideal gas theory and the measured Mach number $M_{s}$ of 1.3 , the incident overpressure $p_{2}-p_{1}$ and reflected overpressure $p_{5}-p_{1}$ are given respectively as $80.6 \mathrm{kPa}$ and $210.9 \mathrm{kPa}$, which is in good agreement with the corresponding pressures in Fig. 4 and indicates that the pressure recordings in this study are reliable.

It is well known that the shock tube technique often replicates some of the characteristics of a pressure loading from an unconfined far-field airblast detonation, i.e. steep rise time and a relatively long decay. This is confirmed by the theory and experiments by Kingery and Bulmash [5], where the reflected overpressure and impulse generated in the shock tube experiment are found to be equivalent to a spherical airburst of $W=1967 \mathrm{~kg}$ TNT at a standoff distance of $R=38 \mathrm{~m}$. Using the Hopkinson-Cranz scaling law $\left(Z=R / W^{1 / 3}\right)$ the blast loading can be categorized with respect to the scaled distance, which is $Z=3.0 \mathrm{~m} / \mathrm{kg}^{1 / 3}$ in this experiment. The experiment may therefore be defined as a far-field airblast [10].

Figure 5 shows an example of synchronized loading and response histories during the experiment. The figure also include a selection of corresponding DIC images in terms of $3 \mathrm{D}$ topography maps, contours of the transverse displacement field and deformation profiles at characteristic times. It is seen that the response is typical for blast loaded plates, i.e. the plate experience large permanent deformations before it vibrates at its new equilibrium position. The DIC images also show plastic hinges traveling from the supports toward the centre of the plate, determining the deformed shape of the plate which is similar to a global dome (Figs. 5c-e). Moreover, the ratio of the positive duration $t_{+}$of the pressure pulse and the natural period of vibration $T_{n}$ for the target plate may be used to characterize the loading (see e.g. [11]) as one 

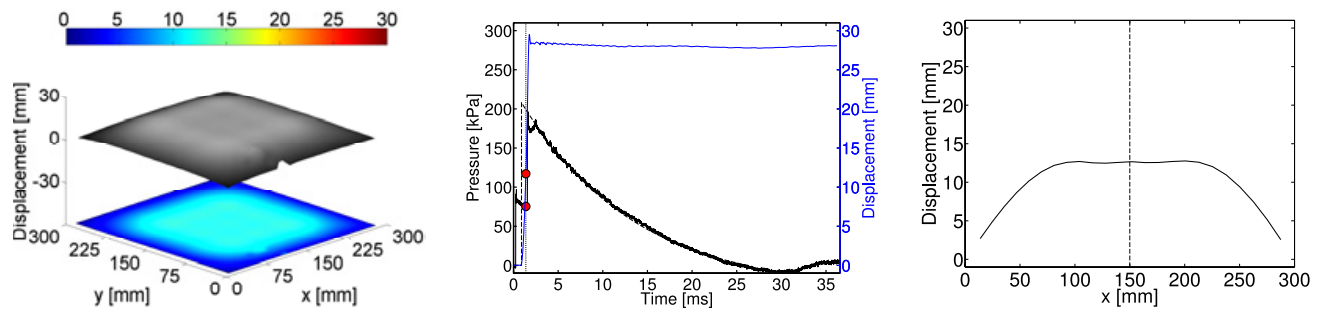

(a) $\mathrm{t}=1.38 \mathrm{~ms}$
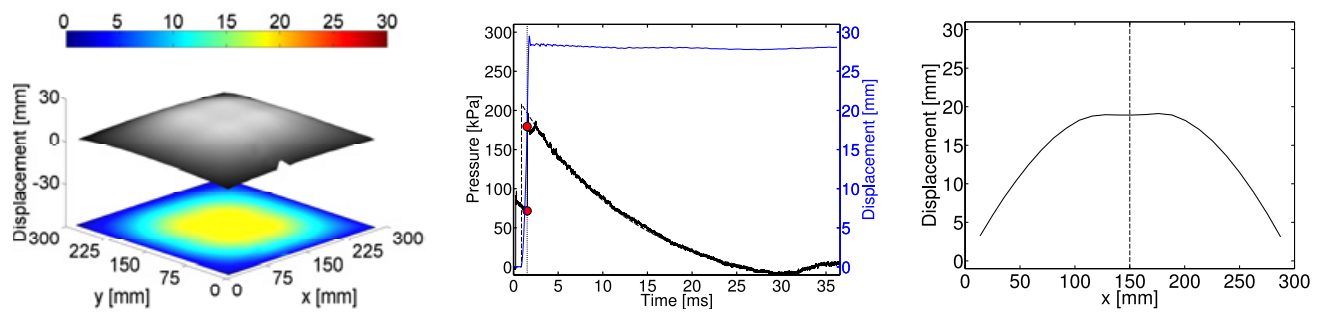

(b) $\mathrm{t}=1.52 \mathrm{~ms}$
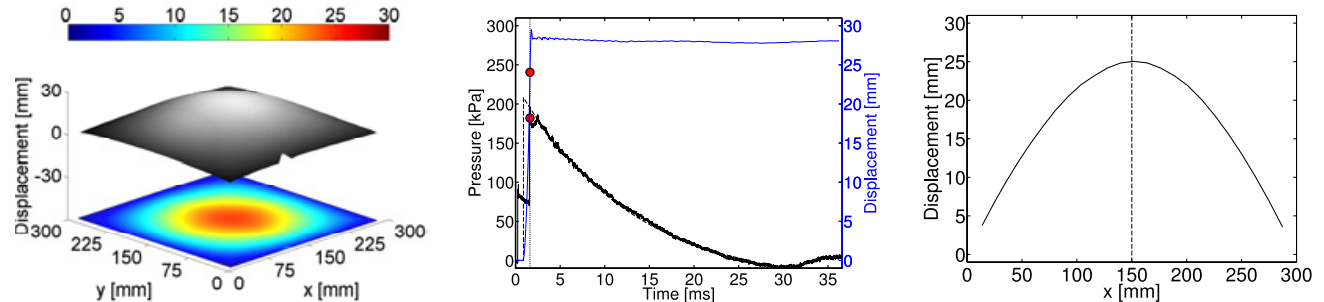

(c) $\mathrm{t}=1.62 \mathrm{~ms}$
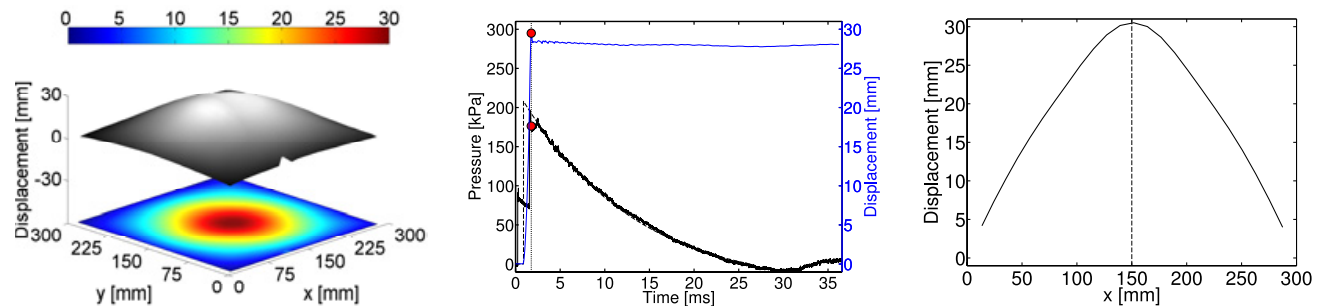

(d) $\mathrm{t}=1.76 \mathrm{~ms}$
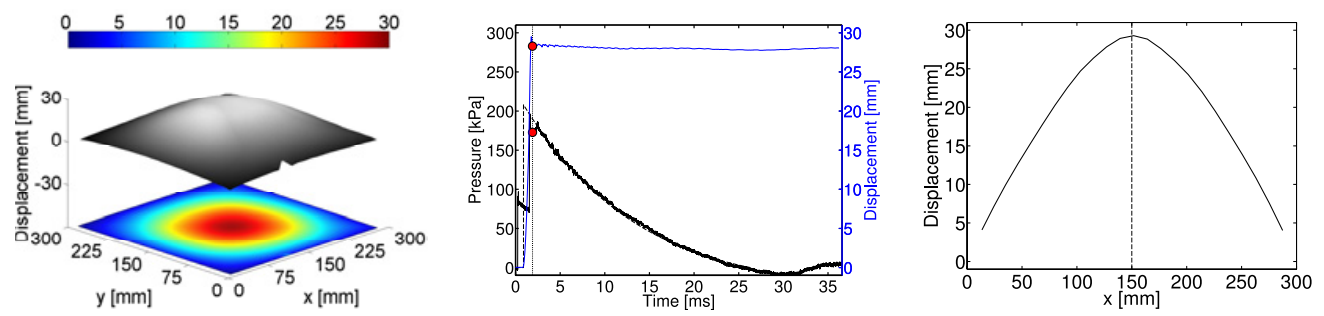

(e) $\mathrm{t}=1.90 \mathrm{~ms}$

Figure 5. A selection of the recorded DIC images and synchronized data from the shock tube experiment.

of the following domains, i.e.

$$
\begin{aligned}
& \frac{t_{+}}{T_{n}}<0.064 \quad \text { (Impulsive domain) } \\
& 0.064<\frac{t_{+}}{T_{n}}<6.4 \quad \text { (Dynamic domain) } \\
& 6.4<\frac{t_{+}}{T_{n}} \quad \text { (Quasi-static domain) }
\end{aligned}
$$

Using the properties of an equivalent SDOF model of a square plate with fixed boundary conditions as given by e.g. Biggs [12], the natural period of vibration is found to be $13.41 \mathrm{~ms}$ for the plate used in this study. Thus, based on the ratio in Table 2, the loading can be classified as dynamic which indicates that the response is significantly influenced by the profile of the load history. 


\section{Concluding remarks}

The experiment showed that the shock tube is capable of recreating a loading similar to that of an unconfined far-field airblast, and the recorded incident Mach number $M_{s}$ shows that the pressure measurements were in good agreement with the idealized gas theory.

This indicates that the shock tube can be used to investigate the material effects, dynamic response, fluidstructure interaction problems, and typical failure modes of structures exposed to blast loading. Suggesting that blast loading may be generated in the shock tube as a cost-effective, easily controllable alternative to explosive detonations. Such experiments are useful for improving the understanding of the response of structures and materials subjected to unintentional external loads, and can be used to compare materials and structures loaded under similar conditions.

Financial support from the Structural Impact Laboratory (SIMLab), Centre for Research-based Innovation (CRI) at the Norwegian University of Science and Technology, is gratefully appreciated. The authors would also like to express their gratitude to Mr. Trond Auestad and Mr. Tore Wisth from CRI-SIMLab for their contributions during the experimental work.

\section{References}

[1] A. G. Hanssen, L. Enstock and M. Langseth, International Journal of Impact Engineering 27, page 593-618 (2002)
[2] T. Børvik, A. G. Hanssen, S. Dey, H. Langberg and M. Langseth, Engineering Structures 30, page 1605-1620 (2008)

[3] T. Børvik, A. Burbach, H. Langberg and M. Langseth, Engineering Structures 30, page 1621-1631 (2008)

[4] Department of Defense (DoD), Structures to Resist the Effects of Accidental Explosions (Report No. UFC 3-340-02, Washington, D.C.: Department of Defense, 2008)

[5] C. N. Kingery, G. Bulmash, Defence Technical Information Center, Ballistic Research Laboratory, Aberdeen Proving Ground, Maryland (1984)

[6] J. A. Zukas, T. Nicholas, H. F. Swift, L. B. Greszczuk and D. R. Curran Impact Dynamics (Wiley \& Sons, New York, USA, 1982) page 316-320

[7] D. K. Christoulis, S. Guetta, V. Guipont and M. Jeandin, Journal of Thermal Spray Technology 20, page 523-533 (2011)

[8] D. Cormie, G. Mays and P. Smith, Blast Effects on Buildings, 2nd edn. (Thomas Telford, London, UK, 2009) page 39-41

[9] A. G. Gaydon and I. R. Hurle, The Shock Tube in High-Temperature Chemical Physics (Chapman and Hall Ltd., London, UK, 1963) page 9-28

[10] D. O. Dusenberry, Handbook for Blast Resistant Design of Buildings (Wiley \& Sons Inc., New Jersey, USA, 2010) page 246-248

[11] W. E. Baker, P. A. Cox, P. S. Westine, J. J. Kulesz and R. A. Strehlow Explosion Hazards and Evaluation (Elsevier, Amsterdam, 1983) page 273-281

[12] J. M. Biggs, Introduction to Structural Dynamics (McGraw-Hill, New York, 1964) page 214 